\title{
A novel design of physical layer network coding in strong asymmetric two-way relay channels
}

\author{
Hao Wei ${ }^{i^{*}}$, Baoyu Zheng ${ }^{1}$ and Xiaodong $\mathrm{Ji}^{1,2}$
}

\begin{abstract}
The quality of two-way links is always asymmetric in practical two-way relay channels (TWRC) and therefore, the capacity of TWRC will be limited by the weaker link. An asymmetric modulation scheme, with physical layer network coding, was presented in order to improve the transmission reliability in TWRC. This makes full use of the stronger link to improve the overall transmission rate and also ensures the reliability of the weaker link. The simulation results show that the proposed asymmetric modulation scheme in the case of strongly asymmetric channels, compared to the symmetric transmission, enhances the system capacity significantly and also guarantees the system reliability.
\end{abstract}

Keywords: Network coding; Asymmetric modulation; Two-way relay channel; Subset constellation

\section{Introduction}

Network coding $(\mathrm{NC})$ was originally proposed to improve the performance of multicast throughput in wired networks by Ahlswede et al. in 2000 [1]. Recently, the broadcast nature of wireless channels has attracted a lot of research activities on the application of $\mathrm{NC}$ in wireless networks.

The two-way relay channel (TWRC) is a typical scenario in wireless communications. Physical layer network coding (PLNC) was proposed to improve the throughput of TWRC [2], which maps the superimposition of the signals received simultaneously to a digital bit stream. PLNC improves the system performance by making use of the interference, instead of avoiding it. PLNC can further be classed into two categories - PNCF (PNC over finite field) and PNCI (PNC over infinite field) - according to whether the network coding field adopted is finite or infinite [3]. The capacity of TWRC with PLNC is higher than the traditional communication strategies [4,5]. The design of modulation suited for TWRC with PLNC can be BPSK or QPSK [6], and an unconventional 5-ary modulation which is optimized according to the channel condition [7]. In addition, PLNC can be combined with channel code

\footnotetext{
* Correspondence: nupt2006@126.com

${ }^{1}$ Institute of Signal Processing and Transmission, Nanjing University of Posts and Telecommunications, Nanjing 210003 Jiangsu, People's Republic of China Full list of author information is available at the end of the article
}

to improve bit error rate (BER) performance of the system [8].

The research works reported above are all based on the same assumption that the transmission rate of two end nodes is symmetric. In practical TWRC, however, the quality of two-way links is always asymmetric. Therefore, the capacity of TWRC will be limited by the weaker link. Thus, in the symmetric rate transmission of TWRC, in order to ensure the reliability of the weaker link, the stronger link has to transmit and receive with low-order modulation as same as the weaker link. For this reason, the stronger link does not take advantage of its good channel conditions to improve the overall transmission rate, which lowers the validity.

Asymmetric modulation is a method to realize the asymmetric rate transmission. The power matching ratio of the two end nodes in TWRC is corresponding to the performance of symbol error rate in the multiple access phase $[9,10]$. In the broadcast phase of TWRC, under the same BER constraint, the weaker link can decode at lower signal noise ratio (SNR) compared to the stronger link by exploiting a priori bit information in each transmit symbol [11].

This paper investigates the asymmetric rate transmission both in the multiple access phase and broadcast phase of the two-phase TWRC by designing an asymmetric modulation scheme with PLNC. The simulation results show that the proposed scheme not only 
improves the transmission validity by increasing the system capacity but also guarantees the transmission reliability.

The rest of this paper is organized as follows. Section 2 introduces the system model. Section 3 presents the new scheme of asymmetric modulation with PLNC. Section 4 analyzes the performance of the asymmetric modulation scheme. Simulation experiments and performance comparisons between the symmetric transmission mode and the proposed scheme will be discussed in Section 5. Finally, Section 6 concludes this paper.

\section{System model}

We consider the two-phase TWRC system as shown in Figure 1, in which two independent end nodes $n_{1}$ and $n_{2}$ exchange information with each other via the relay node $n_{\mathrm{R}}$. As illustrated in Figure $1, B_{i}, i \in\{1,2, R\}$ denotes the bit information of node $n_{i}$ which is modulated to symbol $X_{i}$. The coefficient $h_{i j}$, $i, j \in\{1,2, R\}$ is the complex pathloss coefficient for the channel from $n_{i}$ to $n_{j}$. $W_{i}$ is the noise at $n_{i}$ to be zero-mean complex Gaussian variable with variance $\sigma_{i}^{2} . P_{i}$ is the transmitting power of $n_{i}$. In this paper, the following assumptions are made: (1) all nodes operate in a half-duplex manner, i.e., a node cannot transmit and receive simultaneously; (2) symbol-level time synchronization is assumed; (3) there is no direct link between $n_{1}$ and $n_{2}$; (4) channel coefficient is quasi-static and possess reciprocity, i.e., $h_{1 \mathrm{R}}=h_{\mathrm{R} 1}=h_{1}, h_{2 \mathrm{R}}=h_{\mathrm{R} 2}=h_{2}$; (5) nodes can perfectly estimate the channel state information (CSI) to realize the phase synchronization and amplitude pre-equalization; (6) the energy of symbol $X_{i}$ is normalized to unit 1 ; (7) power constrains $P_{1}+P_{2}=1, P_{\mathrm{R}}=1$.

The process of information exchange comprises of two phases:

1. Multiple access phase (MAC). First, $n_{1}$ and $n_{2}$ modulate $B_{1}$ and $B_{2}$ respectively to $X_{1}$ and $X_{2}$. Second, $n_{1}$ and $n_{2}$ transmit $X_{1}$ and $X_{2}$ to the relay node $n_{\mathrm{R}}$ simultaneously. Then $n_{\mathrm{R}}$ demodulates the superimposition of the signal $Y_{\mathrm{R}}$ to $B_{\mathrm{R}}=B_{1} \oplus B_{2}$.

2. Broadcast phase (BRC). The relay node $n_{R}$ modulates $B_{\mathrm{R}}$ to $X_{\mathrm{R}}$ and broadcasts it to $n_{1}$ and $n_{2}$, simultaneously. Then $n_{1}$ demodulates $Y_{1}$ to $B_{\mathrm{R}}$ and get the bit information of $n_{2}$ by using exclusive or (XOR) operation $B_{2}=B_{1} \oplus B_{\mathrm{R}}$. Similarly,

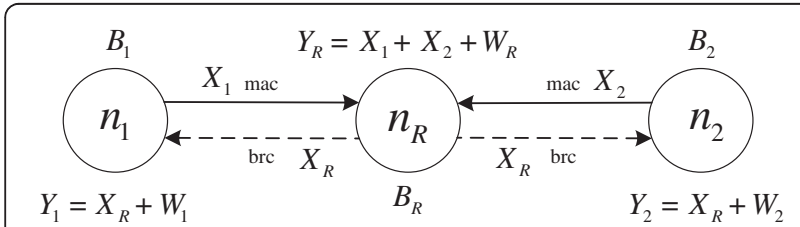

Figure 1 System model of TWRC. $n_{2}$ demodulates $Y_{2}$ to $B_{\mathrm{R}}$ and get the bit information of $n_{1}$ by using XOR operation $B_{1}=B_{2} \oplus B_{\mathrm{R}}$.

So, $n_{1}$ and $n_{2}$ can finish the information exchange only by two phases. The importance of the information exchange is the design of modulation and demodulation at the relay node $n_{\mathrm{R}}$. In multiple access phase, $n_{\mathrm{R}}$ is supposed to realize the demodulation of $Y_{\mathrm{R}} \rightarrow B_{\mathrm{R}}=B_{1} \oplus B_{2}$. In broadcast phase, $n_{\mathrm{R}}$ has to realize the modulation of $B_{\mathrm{R}} \rightarrow X_{\mathrm{R}}$. The performance of the system depends on the design at $n_{R}$, which is a key point in this paper.

\section{Asymmetric rate transmission}

The design of the modulation and demodulation for $n_{1}$, $n_{2}$, and $n_{\mathrm{R}}$ will be presented in this section to realize the asymmetric rate transmission both in the multiple access phase and broadcast phase. For simplicity and without loss of generality, we assume $\sigma_{h_{1}}^{2}>\sigma_{h_{2}}^{2}$ (respectively denote the variances of $h_{1}$ and $h_{2}$ ) which means that the stronger link $C_{1}$ (the channel between $n_{1}$ and $n_{\mathrm{R}}$ ) has better quality than the weaker link $C_{2}$ (the channel between $n_{2}$ and $n_{\mathrm{R}}$ ).

\subsection{Design of asymmetric modulation}

The asymmetric modulation is proposed by utilizing the asymmetric channel quality to make $n_{1}$ and $n_{2}$ transmit and receive at different rates. For simplicity and clarity, QPSK and 16QAM will be as examples to be depicted for the design of the asymmetric modulation, which are respectively $2 \mathrm{bit} / \mathrm{symbol}$ and $4 \mathrm{bit} / \mathrm{symbol}$.

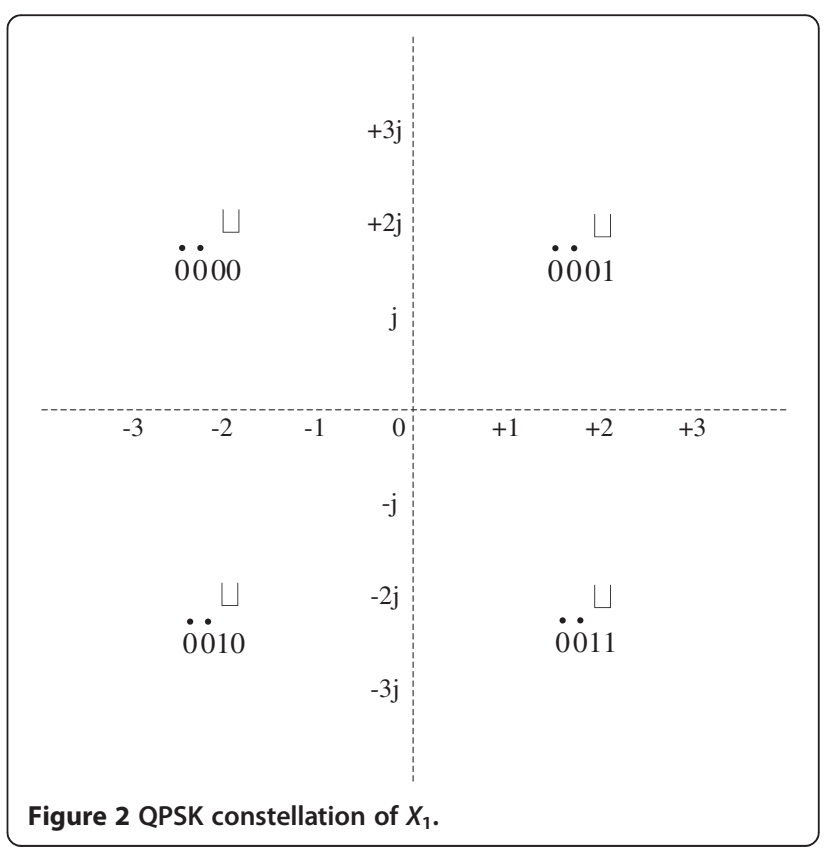




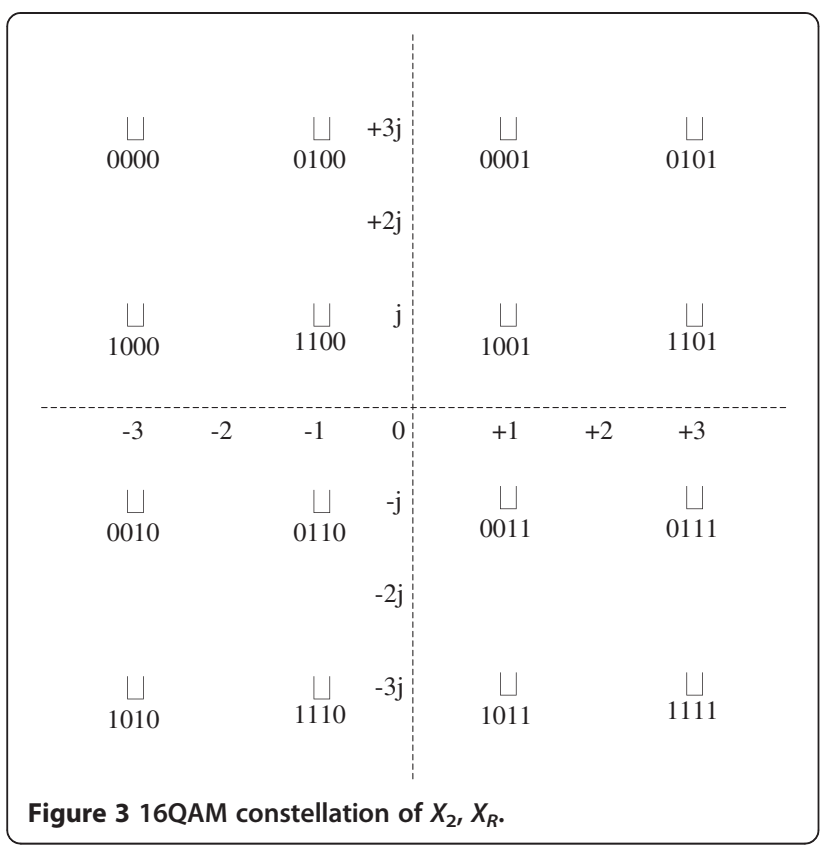

\subsubsection{MAC phase}

Before transmitting, $n_{1}$ first inserts two dummy zeros '00' for each two bits in $B_{1}$ to obtain $\dot{B}_{1}$ making '00,01, 10 , and '11' become '0000,0001, 0010 ', and'0011' (these dummy zeros contain no information and their positions are fixed and known to both $n_{1}$ and $n_{2}$ ); Then $n_{1}$ modulates $\dot{B}_{1}$ to $X_{1}$ by QPSK, and $n_{2}$ modulates $B_{2}$ to $X_{2}$ by 16QAM (the constellation of $X_{1}$ and $X_{2}$ are shown in Figures 2 and 3. Finally, $n_{1}$ and $n_{2}$ transmit $X_{1}$ and $X_{2}$ to $n_{\mathrm{R}}$, respectively. So in the same symbol period, $n_{1}$ transmits two-bit information while $n_{2}$ transmits four bit information.

As illustrated in Figures 2 and 3, the stronger link $C_{1}$ transmits signals by low-order modulation (QPSK), while the weaker link $C_{2}$ transmits signals by high-order modulation (16QAM). This kind of design might be opposite to our straight thinking, but it lets the system make use of the stronger link $C_{1}$ to improve the overall transmission rate and also guarantee the transmission reliability of the weaker link $C_{2}$ in the BRC phase (the specific statement is next). When the transmitting power ratio is $P_{\mathrm{QPSK}}: P_{16 \mathrm{QAM}}=4: 5$, the BER performance of the QPSK-16QAM superimposition signals is optimal in the MAC phase, which is demonstrated in [9]. Note that there are 36 points in the QPSK-16QAM constellation as shown in Figure 4.

\subsubsection{BRC phase}

In the BRC phase, $n_{\mathrm{R}}$ first modulates $B_{R}$ to $X_{R}$. Then $n_{\mathrm{R}}$ broadcasts $X_{\mathrm{R}}$ to $n_{1}$ and $n_{2}$. The constellation of $X_{\mathrm{R}}$ is shown in Figure 3, which is same as the constellation of $X_{2}$ in the MAC phase.

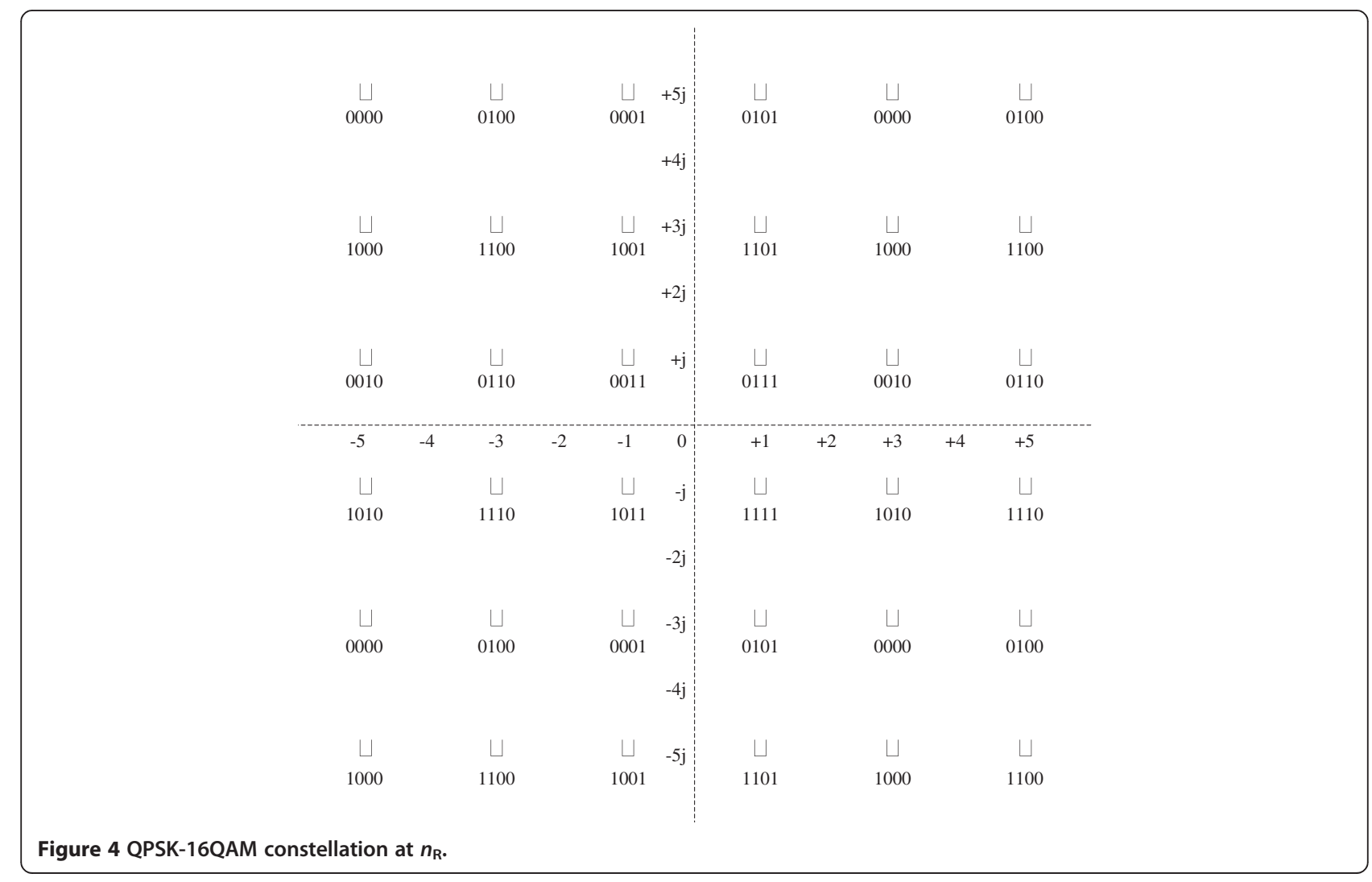


Due to the fore 2 bit ' 00 ' in each 4 bit of $\dot{B}_{1}$ which is inserted by $n_{1}$ as the dummy zeros, the fore 2 bits of $B_{2}$ are kept unchanged in $B_{\mathrm{R}}$ after the XOR operation at $n_{\mathrm{R}}$. So $n_{2}$ can exploit these as a priori information and does not need to decode every bit in $B_{R}$. Node $n_{2}$ can discard the known bits and decode only the latter 2 bits of $B_{R}$ according to the subset of 16QAM constellation (QPSK), which is illustrated in Figure 5. Then $n_{2}$ can obtain the bit information of $n_{1}$ by executing the XOR operation $B_{1}=B_{2} \oplus B_{\mathrm{R}}$.

As illustrated in Figure 5, the constellation used to demodulate signals by $n_{2}$ is QPSK, which is a subset of 16QAM constellation. In this way, the distance of the adjacent points is increased, so the BER performance is improved and the transmission reliability is guaranteed.

By using this scheme, in BRC phase, the signal modulated by high-order modulation (16QAM) is transmitted through the stronger link $C_{1}$ to improve the transmission rate, while the signal modulated by low-order modulation (QPSK, which is the subset of 16QAM constellation) is transmitted through the weaker link $C_{2}$ to ensure the transmission reliability.

\subsection{Symmetric modulation for comparison}

In order to show up the advantages of the proposed asymmetric modulation scheme, we use the symmetric

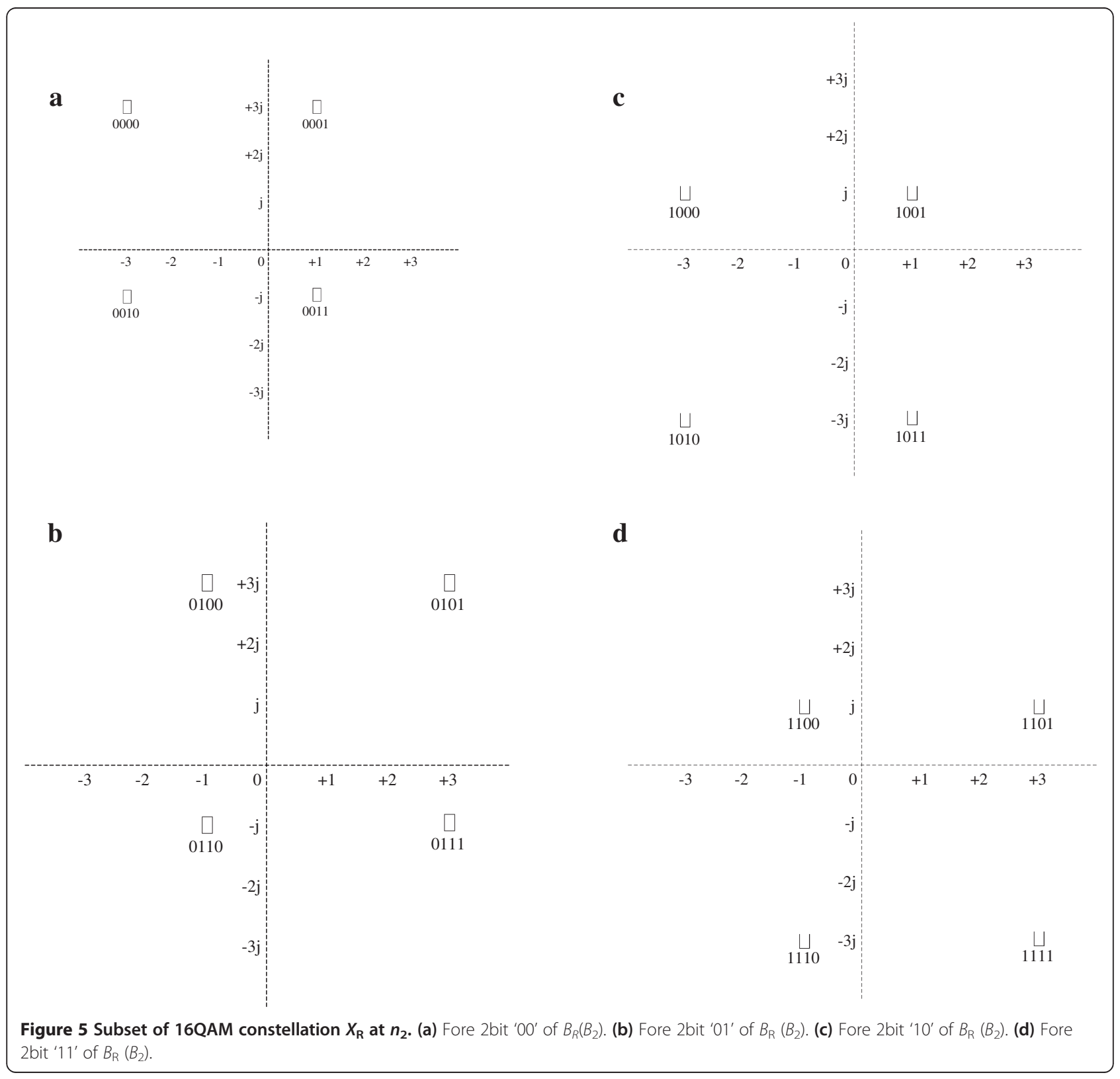


modulation QPSK-QPSK as examples for a simple comparison.

By using symmetric modulation, the stronger link $C_{1}$ has to adopt the same low-order modulation as the weaker link $C_{2}$ to transmit and receive signals. Both $n_{1}$ and $n_{2}$ adopt QPSK modulation to transmit and receive signals, and $n_{\mathrm{R}}$ adopt QPSK modulation to transmit the XOR signals. The constellations are shown in Figures 6 and 7.

\section{Performance analysis}

In this section, we will analyze the performance of the proposed asymmetric modulation and compare it to that of the symmetric modulation. As we known, $C_{1}$ is better than $C_{2}$. Without loss of generality, we assume $\mu>1$. For simplicity, we assume that the noises at the three nodes have the same variance, $\sigma_{1}^{2}=\sigma_{2}^{2}=\sigma_{\mathrm{R}}^{2}=\sigma^{2}$.

\subsection{BER analysis}

\subsubsection{MAC phase}

Here, we use $d_{\min }$ to denote the minimum distance between the adjacent points in the constellation of the superimposition of the signals received at $n_{\mathrm{R}}$ (as shown in Figures 4 and 7). Thus, we have

$$
\begin{array}{ll}
d_{\mathrm{QPSK}-16 \mathrm{QAM} \min }=2 / 3 & \approx 0.667 \\
d_{\mathrm{QPSK}-\mathrm{QPSK} \min }=\sqrt{2} & \approx 1.414
\end{array}
$$

Then the approximation of the BER can be obtained according to $[12,13]$ as

$$
\begin{aligned}
& \text { BER } \\
& \text { QPSK-16QAM } \approx \frac{5}{\log _{2} 16} Q\left(\sqrt{\frac{2}{9} \gamma_{\mathrm{R}}}\right), \\
& \text { BER } R_{\text {QPSK-QPSK }} \approx \frac{3}{\log _{2} 4} Q\left(\sqrt{\gamma_{\mathrm{R}}}\right)
\end{aligned}
$$

Where $\gamma_{R}$ is SNR of the signals received at $n_{R}$.

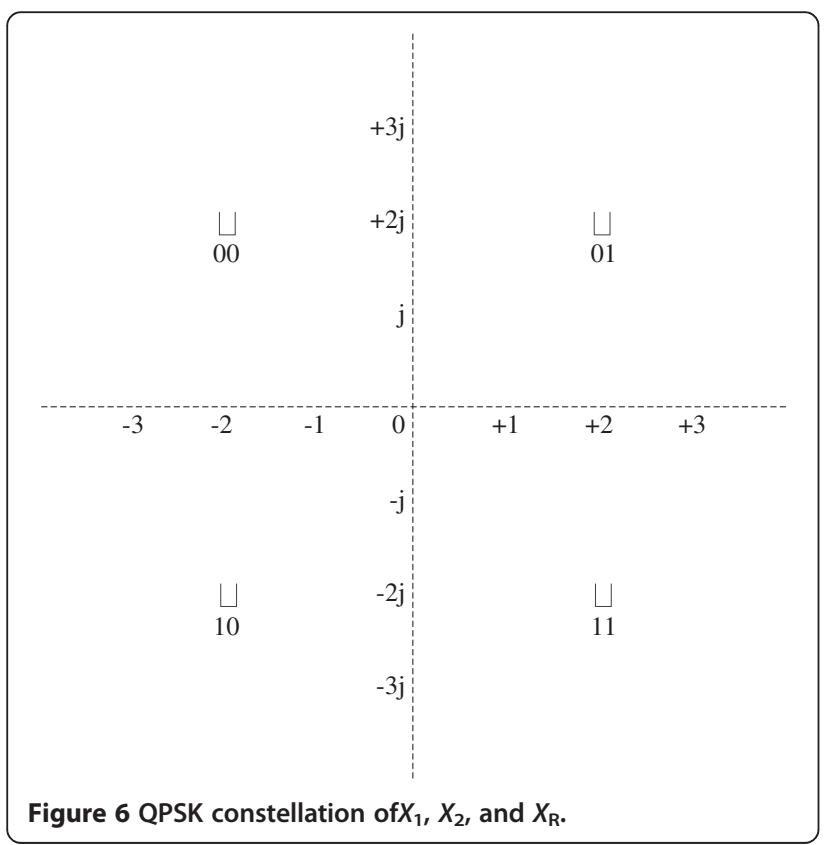

\subsubsection{BRC phase}

Also, we use $d_{\min }$ to denote the minimum distance between the adjacent points in the constellation of the signals received at $n_{1}$ and $n_{2}$ from $n_{\mathrm{R}}$ (as shown in Figures 3, 5, and 6). Then we have

$$
\begin{array}{llll}
d_{n_{1}-16 \mathrm{QAM}_{\text {ASY }} \min }=2 \sqrt{5} / 5 & \approx 0.894 & d_{n_{2}-\mathrm{QPSK}_{\mathrm{ASY}} \min }=4 \sqrt{5} / 5 & \approx 1.789 \\
d_{n_{1}-\mathrm{QPSK}_{\mathrm{SYM}} \min } & =2 & d_{n_{2}-\mathrm{QPSK}_{\mathrm{SYM}} \min } & =2
\end{array}
$$

Further, the approximation of the BER can be obtained according to $[12,13]$ as

$$
\begin{aligned}
& \mathrm{BER}_{n_{1}-16 \mathrm{QAM}} \approx \frac{4}{\mathrm{ASY}} \approx \frac{4}{\log _{2} 16} Q\left(\sqrt{\frac{2}{5} \gamma_{1}}\right) \\
& \mathrm{BER}_{n_{1}-\mathrm{QPSK}_{\mathrm{SYM}}} \approx \frac{2}{\log _{2} 4} Q\left(\sqrt{2 \gamma_{1}}\right)
\end{aligned}
$$

$$
\begin{gathered}
\mathrm{BER}_{n_{2}-\mathrm{QPSK}_{\mathrm{ASY}}} \approx \frac{2}{\log _{2} 16} Q\left(\sqrt{2 \times \frac{4}{5} \gamma_{2}}\right) \\
\mathrm{BER}_{n_{2}-\mathrm{QPSK}_{\mathrm{SYM}}} \approx \frac{2}{\log _{2} 4} Q\left(\sqrt{2 \gamma_{2}}\right)
\end{gathered},
$$

where $\gamma_{1}$ and $\gamma_{2}$ denote SNR of the signals received at $n_{1}$ and $n_{2}$, respectively.

\subsection{Capacity analysis}

According to $[14,15]$, the AWGN channel in MAC phase and BRC phase can be regarded as an equivalent virtual channel $C_{\mathrm{V}}$ shown in Figure 8.

The input of $C_{\mathrm{V}}$ is $X$ and the output is $Y=X+W$, where $W$ is a zero-mean complex Gaussian random variable $W \sim C N\left(0, \sigma^{2}\right), C_{\mathrm{V}}$ is a discrete-input and continuous-output channel according to [15]. The input $X$ comprises symbols selected from a finite and discrete input alphabet $X=x_{k}(k=0,1, \ldots, q-1)$, and the output is continuous $Y=\{-\infty,+\infty\}$. For a given $X$, it follows that $Y$ is a complex Gaussian random variable with mean $x_{k}$ and variance $\sigma^{2}$, that is,

$$
p\left(y \mid X=x_{k}\right)=\frac{1}{\sqrt{2 \pi} \sigma} e^{-\left(y-x_{k}\right)^{2} / 2 \sigma^{2}} .
$$

For any given input sequence $X_{i}(i=1,2, \ldots, n)$, there is a corresponding output sequence of

$$
Y_{i}=X_{i}+W_{i} \quad(i=1,2, \ldots, n) .
$$

The condition that the channel is memoryless can be expressed as

$$
\begin{aligned}
& p\left(y_{1}, y_{2}, \ldots, y_{n} \mid X_{1}=u_{1}, X_{2}=u_{2}, \ldots, X_{n}=u_{n}\right) \\
& \quad=\prod_{i=1}^{n} p\left(y_{i} \mid X_{i}=u_{i}\right) .
\end{aligned}
$$




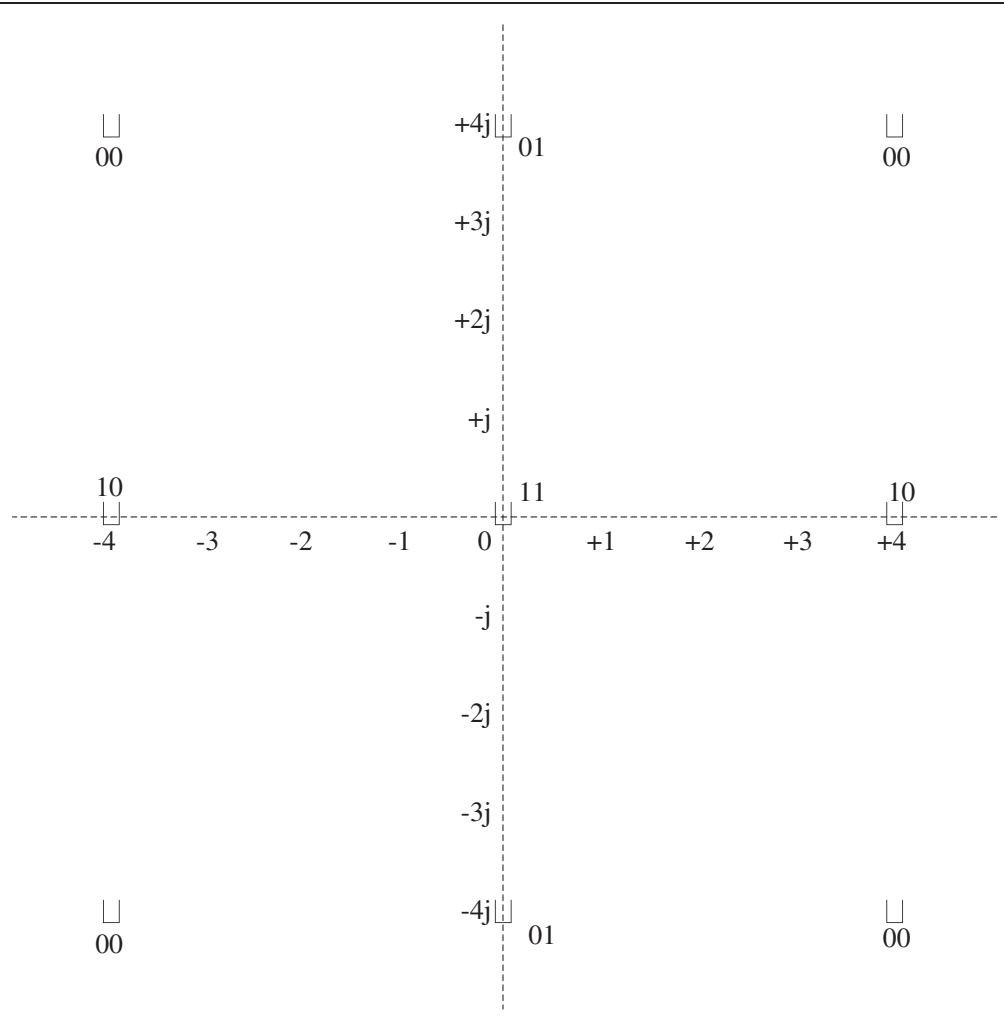

Figure 7 QPSK-QPSK constellation at $n_{\mathrm{R}}$.

So, the capacity of $C_{\mathrm{V}}$ is defined as

$$
\begin{aligned}
C_{\mathrm{V}} & =\max _{p\left(x_{i}\right)} I(X ; Y) \\
& =\max _{p\left(x_{i}\right)} \sum_{i=0}^{q-1} \int_{-\infty}^{+\infty} p\left(y \mid x_{i}\right) p\left(x_{i}\right) \log \frac{p\left(y \mid x_{i}\right)}{p(y)} \mathrm{d} y \\
& =\max _{p\left(x_{i}\right)} \sum_{i=0}^{q-1} \int_{-\infty}^{+\infty} p\left(y \mid x_{i}\right) p\left(x_{i}\right) \log \frac{p\left(y \mid x_{i}\right)}{\sum_{k=0}^{q-1} p\left(y \mid x_{k}\right) p\left(x_{k}\right) \mathrm{d} y .}
\end{aligned}
$$

The input $X$ are equally probable symbols, and the probability $p\left(x_{i}\right)$ can be obtained according to the corresponding modulation constellation. Substituting $p\left(x_{i}\right)$ and Equation 7 into Equation 8, we can obtain the capacity of the equivalent virtual channel $C_{\mathrm{V}}$.

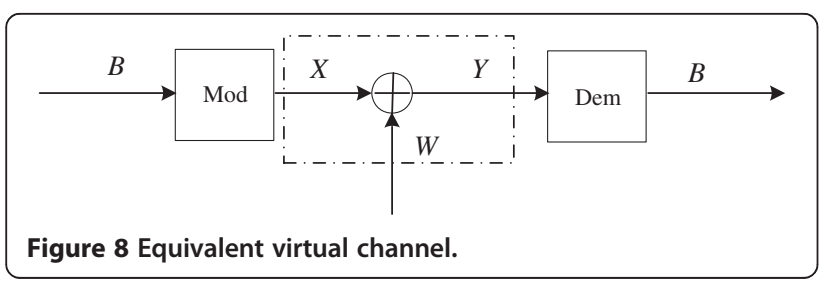

\section{Simulation results}

In this section, simulation results are presented to demonstrate the performance of our proposed scheme and to verify the accuracy of our analytical analysis in Section 4. In the simulation, all the numerical results are calculated with averaging over 10,000,000 packets, and the number of bits contained by each packet is equal to the bits contained by each symbol in the corresponding constellation. For simplicity and without loss of generality, we consider two scenarios of $\mu=4$ and $\mu=8$. The SNR of the stronger link $C_{1}$ is 6 and $9 \mathrm{~dB}$ higher than that of the weaker $\operatorname{link} C_{2}$.

\subsection{BER performance}

Here, the simulation results are presented to demonstrate the BER performance of the asymmetric modulation at $n_{1}$ and $n_{2}$.

Figures 9 and 10 respectively show the BER performance of $n_{1}$ and $n_{2}$ when the asymmetric levels of the two-way links are 6 and $9 \mathrm{~dB}$. As can be seen in Figures 9 and 10, when the asymmetric modulation QPSK16QAM is adopted, although the modulation is different (one is QPSK, another is 16QAM), the BER performance of $n_{1}$ is close to $n_{2}$. Moreover, the closeness of the BER performance for the symmetric modulation QPSK-QPSK is similar with the asymmetric modulation QPSK16QAM. When the asymmetric level of the two-way 


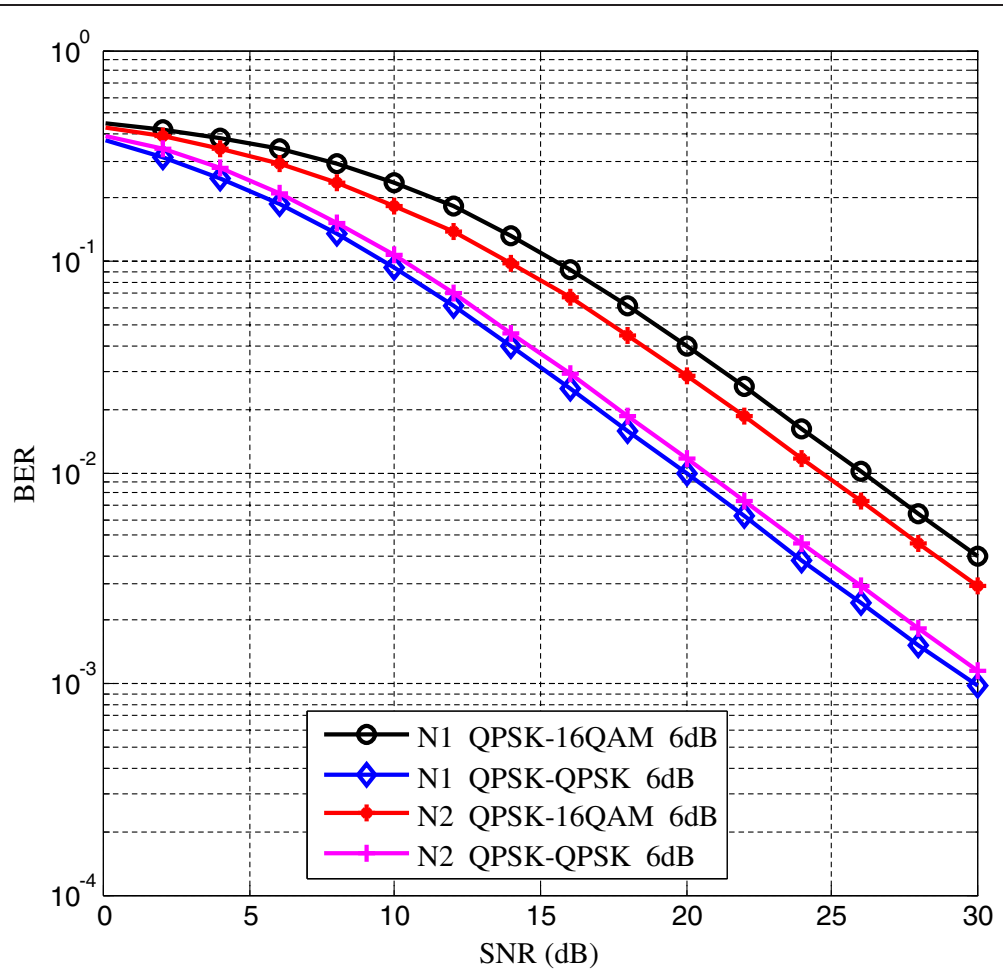

Figure 9 BER performance (asymmetric level is $6 \mathrm{~dB}$ ).

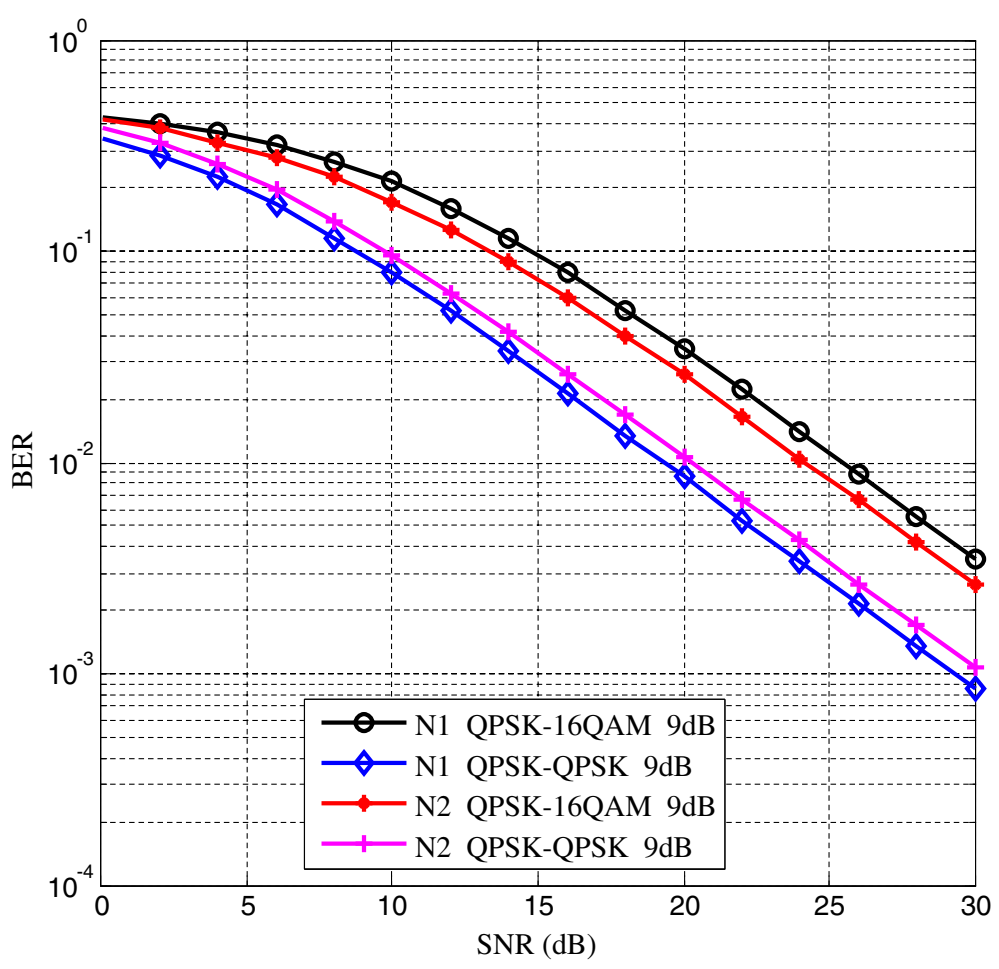

Figure 10 BER performance (asymmetric level is $9 \mathrm{~dB}$ ). 


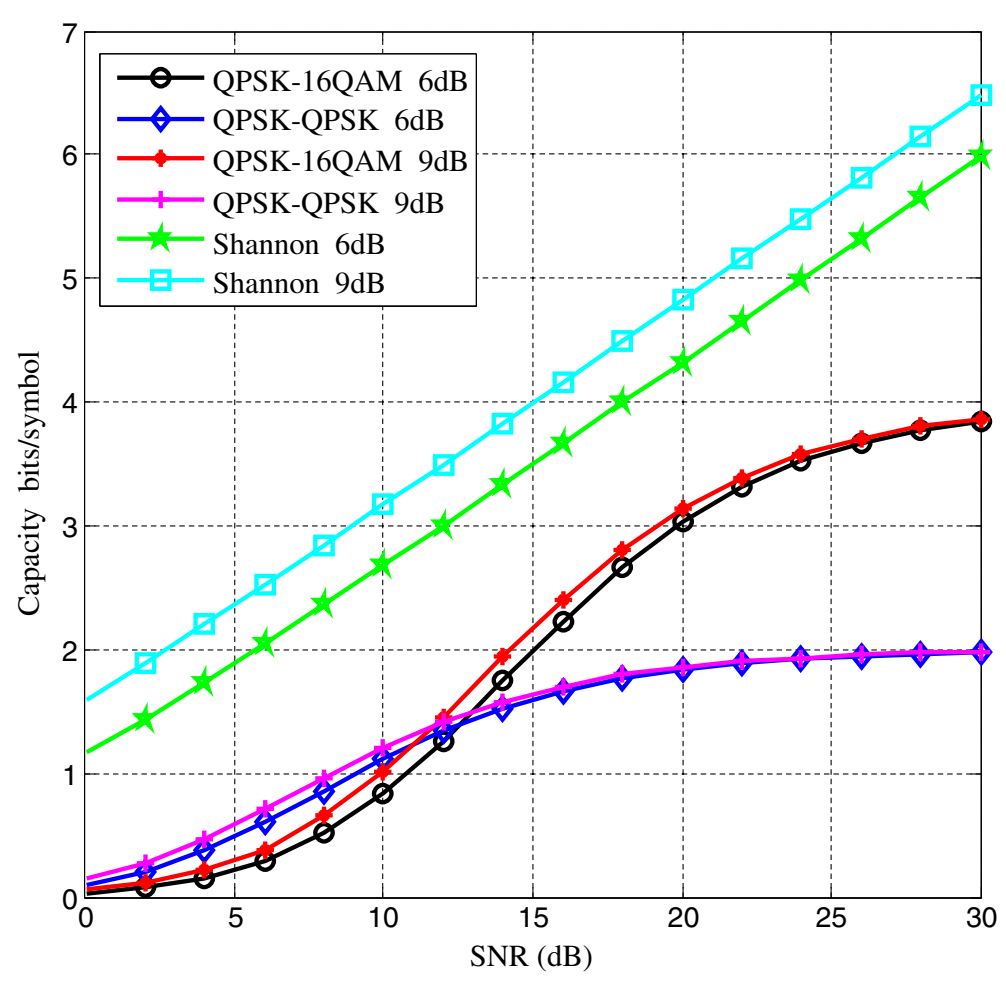

Figure 11 Capacity at $n_{1}$.

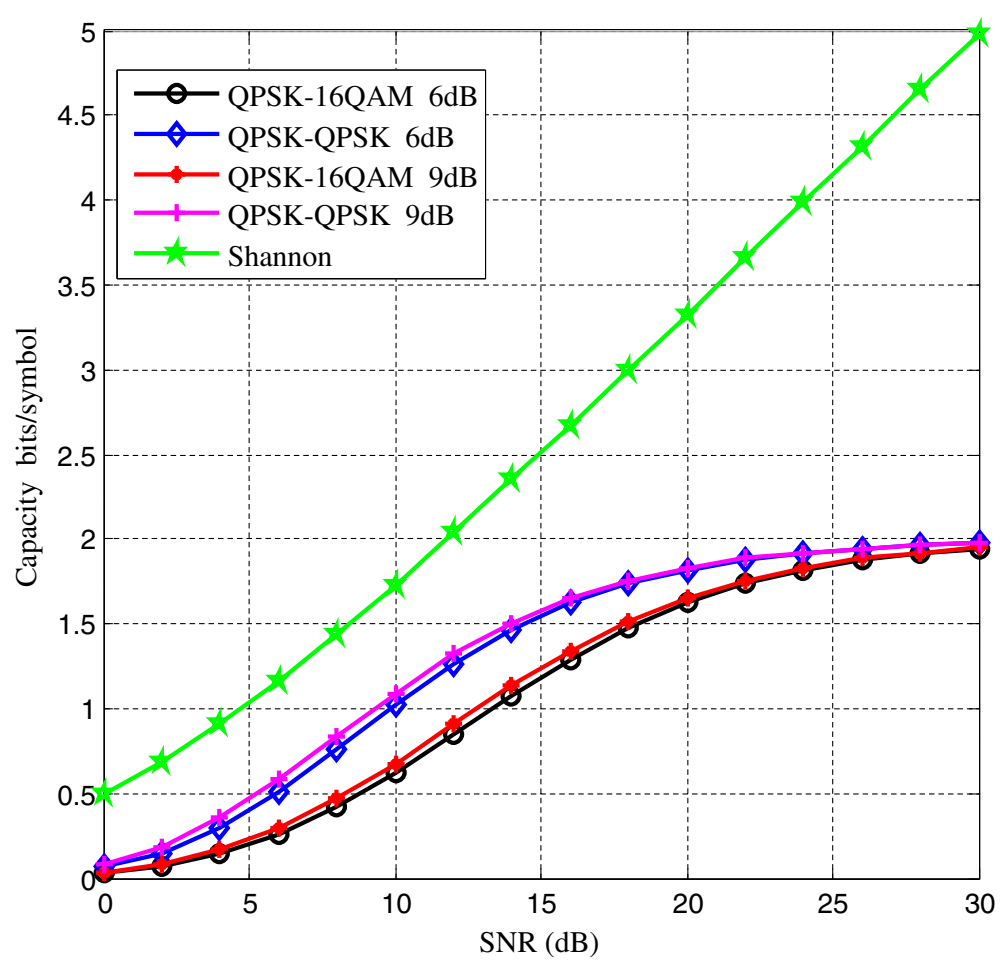

Figure 12 Capacity at $n_{2}$. 


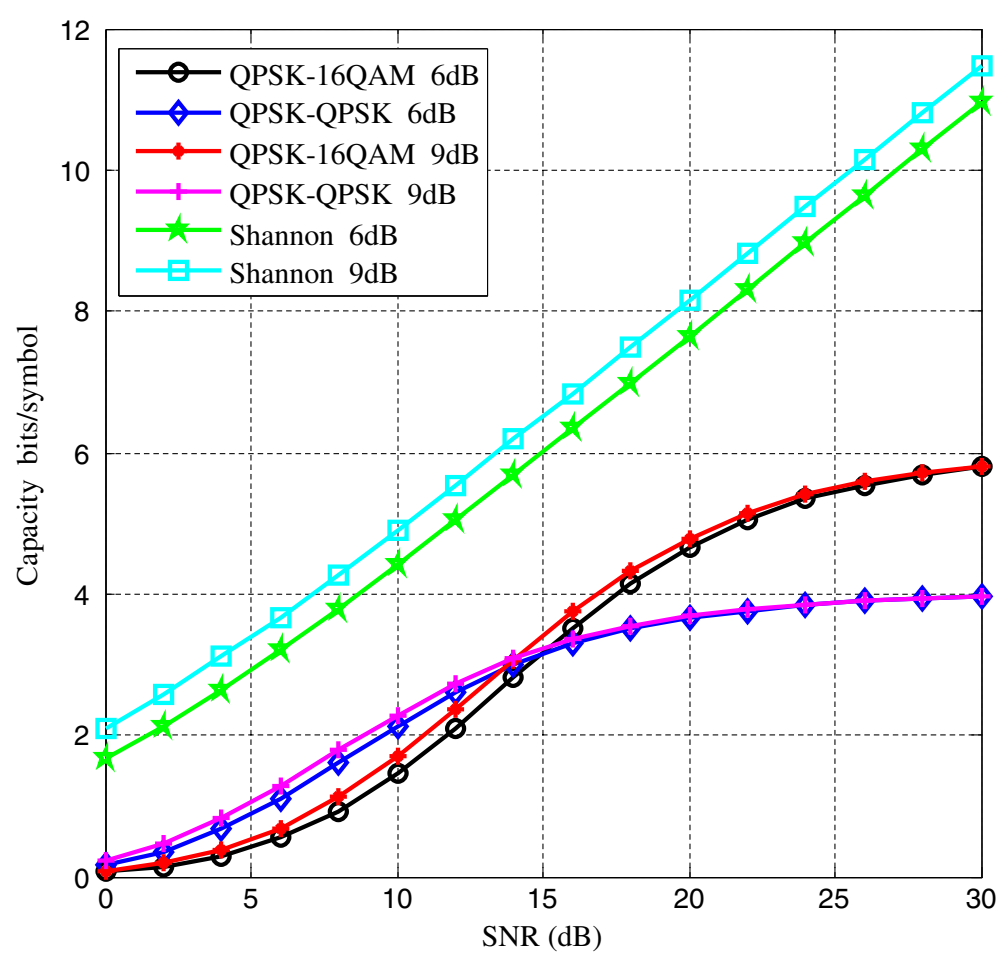

Figure 13 Sum of capacity.

links increases (from 6 to $9 \mathrm{~dB}$ ), the trend remains unchanged.

\subsection{Capacity}

Here, the simulation results are presented to demonstrate the capacity of the asymmetric modulation at the end nodes $n_{1}$ and $n_{2}$. The sum of capacity is also illustrated here.

Figures 11 and 12 respectively show the capacity of $n_{1}$ and $n_{2}$. The $x$-axis is the SNR of $C_{2}$ in the two figures above, which needs to be paid attention to. As shown in Figure 11, when the SNR of the stronger link $C_{1}$ is $6 \mathrm{~dB}$ higher than that of the weaker link $C_{2}$ and the SNR is higher than $13 \mathrm{~dB}$, the capacity of $n_{1}$ by using the asymmetric modulation QPSK-16QAM is higher than by using the symmetric modulation QPSK-QPSK. When the SNR of the stronger link $C_{1}$ is $9 \mathrm{~dB}$ higher than that of the weaker link $C_{2}$, the situation is similar when SNR is higher than $11 \mathrm{~dB}$. As shown in Figure 12, when the channel quality is asymmetric, the capacity of $n_{2}$ by using the asymmetric modulation QPSK-16QAM is close to the capacity of $n_{2}$ by using the symmetric modulation QPSKQPSK.

Figure 13 shows the total capacity of $n_{1}$ and $n_{2}$ when the asymmetric level of the two-way links is 6 and $9 \mathrm{~dB}$. As shown in Figure 13, when the SNR of the stronger link $C_{1}$ is $6 \mathrm{~dB}$ higher than that of the weaker link $C_{2}$ and the SNR is higher than $15 \mathrm{~dB}$, the capacity of $n_{1}$ by using the asymmetric modulation QPSK-16QAM is higher than by using the symmetric modulation QPSKQPSK. When the SNR of the stronger link $C_{1}$ is $9 \mathrm{~dB}$ higher than that of the weaker link $C_{2}$, the situation is similar when SNR is higher than $13 \mathrm{~dB}$. Overall, under higher SNR, compared with the symmetric modulation, the total capacity has been greatly improved by using the asymmetric modulation.

\section{Conclusions}

In this paper, an asymmetric modulation scheme with PLNC in TWRC is proposed, which aims to improve both the validity and reliability in two-way relay transmissions. The proposed asymmetric modulation scheme realized the asymmetric rate transmission both in MAC phase and BRC phase of TWRC. In MAC phase, the BER performance at the relay is improved. In BRC phase, the capacity is boosted by making full use of the stronger link, and the BER performance is guaranteed by exploiting a priori bit information to demodulate for the weaker link. We derived the approximated BER expressions for the scheme proposed, which were also demonstrated by simulation experiments. Through the comparisons of the symmetric modulation scheme, it is found that by using the proposed asymmetric modulation scheme, the total capacity is improved significantly under the asymmetric level of the two-way links. 
In addition, as well-known channel coding possesses the correcting ability and can improve the BER performance further, combining channel coding, network coding, and modulation for asymmetric transmissions in TWRC will be our future researches.

\section{Competing interests}

The authors declare that they have no competing interests.

\section{Acknowledgement}

This work was supported by the National Natural Science Foundation of China under No. 61271240

\section{Author details}

'Institute of Signal Processing and Transmission, Nanjing University of Posts and Telecommunications, Nanjing 210003 Jiangsu, People's Republic of China. ${ }^{2}$ Key Lab of Broadband Wireless Communication and Sensor Network Technology, Nanjing University of Posts and Telecommunications, Ministry of Education, Nanjing 210003 Jiangsu, People's Republic of China.

Received: 13 December 2012 Accepted: 28 May 2013

Published: 17 June 2013

\section{References}

1. R. Ahlswede, N. Cai, S.Y. Li, R.W. Yeung, Network information flow. J. IEEE Trans. Inform. Theor.) 46(4), 1204-1216 (2000)

2. S. Zhang, S. Liew, P.L. Patrick, Proceedings of the 12th Annual International Conference on Mobile Computing and Networking, Los Angeles, 24-29 September 2006 (ACM, New York, 2006), pp. 358-365

3. S. Zhang, S. Liew, L. Lu, Physical layer network coding schemes over finite and infinite fields, in Global Telecommunications Conference, IEEE GLOBECOM 2008, 30 November-4 December 2008 (IEEE Communication Society, New Orleans, 2008), pp. 1-6

4. S. Zhang, S. Liew, H. Wang, X. Lin, Capacity of two-way relay channel. Access Netw.: Inst. Comput. Sci., Soc. Inform. Telecom. Eng. 37(2), 219-231 (2010)

5. P. Popovski, H. Yomo, Physical network coding in two-way wireless relay channels, in IEEE International Conference on Communications, ICC 2007, Glasgow, 24-28 June 2007 (IEEE Press, Glasgow, 2007), pp. 707-712

6. K. Lu, S. Fu, Y. Qian, H.-H. Chen, SER performance analysis for physical layer network coding over AWGN channels, in Global Telecommunications Conference, IEEE GLOBECOM 2009, Honolulu, 30 November-4 December 2009 (IEEE Communication Society Press, Honolulu, 2009), pp. 1-6

7. K.A. Toshiaki, P. Popovski, V. Tarokh, Optimized constellations for two-way wireless relaying with physical network coding. EEE J. Sel. Area Comm. 27(5), 773-787 (2009)

8. S. Zhang, S. Liew, Channel coding and decoding in a relay system operated with physical-layer network coding. EEE J. Sel. Area Comm. 27(5), 788-796 (2009)

9. X. Zhang, Y. Li, J. Lin, G. Li, Z. He, On performance of judging region and power allocation for wireless network coding with asymmetric modulation, in IEEE Vehicular Technology Conference, VTC2011-Fall, San Francisco,5-8 September 2011 (IEEE Press, San Francisco, 2011), pp. 1-5

10. X. Zhang, Y. Li, J. Lin, J. Di, R. Han, Asymmetric network coding in two-way relay channels for cooperative communications: SER performance and power matching, in IEEE International Symposium on Wireless Personal Multimedia Communications, WPMC 2011, Brest, 3-7 October2011 (IEEE Press, Brest, 2011), pp. 1-5

11. J. Zhao, M. Kuhn, A. Wittneben, G. Bauch, Asymmetric data rate transmission in two-way relaying systems with network coding, in IEEE International
Conference on Communications, ICC 2010, Cape Town, May 23-27, 2010 (IEEE Press, Cape Town, 2010), pp. 1-6

12. C. Fan, L. Cao, The Principle of Communications (National Defense Industry Press, Beijing, 2008)

13. A. Goldsmith, Wireless Communications (Cambridge University Press, Cambridge, 2005)

14. Z. Chen, B. Zheng, X. Ji, X. Xiao, An improved joint design of physical layer network coding and channel coding based on trellis coded modulation in two-way relay channel. J. Electron. Inform. Technol. 33(11), 2594-2599 (2011)

15. J. Proakis, Digital Communications (McGraw-Hill, New York, 2000)

doi:10.1186/1687-1499-2013-166

Cite this article as: Wei et al:: A novel design of physical layer network coding in strong asymmetric two-way relay channels. EURASIP Journal on Wireless Communications and Networking 2013 2013:166.

\section{Submit your manuscript to a SpringerOpen ${ }^{\circ}$ journal and benefit from:}

- Convenient online submission

- Rigorous peer review

- Immediate publication on acceptance

- Open access: articles freely available online

- High visibility within the field

- Retaining the copyright to your article

Submit your next manuscript at $\gg$ springeropen.com 